\section{DROPS AND SPLASHES. ${ }^{\mathrm{T}}$}

TH E few who have access to the 'Transactions of the Royal Society, and who remember the first presentation of Prof. Worthington's beautiful photographs illustrating the successive movements that occur in the phenomenon of the splash of a drop, and some proportion of the many who may have seen his two articles on the subject in Pearson's Magazine, will welcome the appearance of the fascinating quarto volume entitled "A Study of Splashes." Not only will their recollection of an interesting research be revived, but the more perfectly executcd and more numerous and complete series of photographs here presented will show the phenomena in all their original beauty as displayed on the lantern screens at the Royal Institution and elsewhere.

Besides showing the results and explaining the interesting cooperation of the forces of dynamics and of surface tension which have given rise to the phenomena, Prof. Worthington has given very full details of his method so that many who can extemporise physical apparatus will be able to follow him, and so to investigate the same or analogous movements.

As a series of twelve or twenty successive photographs illustrating the movements of the liquid which occur in a small fraction of a second cannot at present be taken from a single falling drop or splash, but each requires a new drop to be photographed at a different stage, predetermined in time within one or two thousands of a second, it is essential that so far as is possible the phenomena should be exactly repeated, and that the initial conditions should be identical. The method by which liquid is allowed to drop or balls to fall with sufficient exactness is illustrated and described, and to this no further reference need be made in the present notice. The more interesting part of the apparatus is that in which, the ball or drop having been liberated, an illuminating electric spark is formed as many thousandths of a second after the first contact of the splash as may have been determined, as also is the means of utilising the light of the spark for the purpose of obtaining a shaded picture. This is illustrated on $\mathrm{p}$. 7. The action depends upon the equality of speed of two falling bodies, one the drop or ball which will make the splash, and the other a conducting ball which in its fall will pass close to two nobs forming part of an electric circuit. This circuit comprises the two Leyden jars of an electric influence machine, the nobs in question and the illuminating spark gap, the two nobs being connected with the charged insides, and the terminals of the illuminating spark-gap with the uncharged outsides of the jars. The conducting ball as it passes close to the nobs discharges the circuit, and a spark is formed at the illuminating spark-gap. According as an electromagnetic trigger which liberates the conducting ball is set higher or lower, the time which will elapse after the breaking of the magnetic circuit up to the time of the formation of the spark may be made greater or less at will. The drop or ball which makes the splash is liberated in a similar manner, and so it is merely necessary to increase the height of fall of the conducting ball little by little as compared with that of the drop or splashing ball to illuminate the splash at any desired period of its existence; perhaps not merely necessary, as it is also important that the jars should be charged every time to the same potential, otherwise the spark would

1 "A Study of Splashes." By. Prof. A. M. Worthington, C.B., F.R.S. Price $6 s .6 d$. net.

NO. 233.5 , VOL. 78$]$ not be liberated when the conducting ball had in successive falls reached the same place. The spark is in the focus of an illuminating reflector which gives a large field of light as well as a central bright point, and an ordinary camera with a lens to throw an image of the splash upon the plate completes the means by which the phenomenon which exists at the moment of the spark is photographed with all the perfection of light and shade which make the results so beautiful.

Even with all these precautions successive drops are not necessarily exactly alike at their initiation, as very small changes in the electrical conditions of the two circuits and in the form of a liquid drop, if that is used, at its start will make initial differences which the instability of the liquid forms subsequently developed rapidly accentuate. Still, even with these differences in detail the essential characteristics of a series of drops at different stages are, with the precautions mentioned, so nearly identical that the curiously different characters of splashes made in different circumstances can be well followed throughout their existence.

When the under-water phenomena are photographed the field reflector mentioned above is replaced by a lantern condenser, close to which a fine ground sheet of glass is placed so as to give a uniform field of illumination.

It may be interesting to mention that with the apparatus used by Prof. Worthington he was able to obtain sparks the effective duration of which was not more than three millionths $(3 / \mathbf{r}, 000,000)$ of $a$ second. When it is remembered with what extreme rapidity the edge of a liquid film changes its form under capillary forces, it will be seen that a spark of very short duration is essential, and judging by results one sufficiently short has been obtained.

The book is illustrated by eighteen series of photographs, each series representing a succession of events in one type of splash equivalent as nearly as possible to a succession of events in a single splash. The first series is represented by twenty-four successive photographs of a drop of water falling through a space of 40 centimetres into still milk and water, and there is an alternative smaller series. The second shows the fall of water into water, and incidentally the importance of keeping the water into which the drop falls perfectly free from contamination. Two corresponding photographs are shown side by side; in one the water had been skimmed to purify it from the small trace of grease and smoke which the previous falling drop had brought down from the smoked spoon in which it had been supported; in the other the surface was completely renewed by keeping up a gentle supply of pure water so that contanination could be completely removed by gentle overflow. The absence of a multitude of fine ripples in the first contrasts with the closely furrowed surface of the second, as does a patch on the sea into which a sardine tin has been emptied of its oil with the surrounding portions on a day when the surface is black with small ripples.

Succeeding series show the formation of bubbles, the contrast in the splashes formed by smooth and rough spheres, and other phenomena which it is difficult to describe adequately in a notice and without the photographs to refer to. Some of these are illuminated from above only, and some are illuminated by light on a level with the liquid surface, a transparent cell being used in this case so that the underwater phenomena may be seen as weil as those above the surface.

The last series illustrates the effect of the fall of a 
rough sphere into water from a height of $\mathrm{I} 40$ centi- realised unintentionally when a leaking tap allowing metres. Figs. 1, 2, 3, 4, and 5 here reproduced are drops to fall into a vessel full of water is nevertheles from this series, and they serve well to show the so disposed that either succeeding drops are not almost beauty of Prof. Worthington's results, as also the exactly alike or the water into which the drops fall curious formation of a liquid jet within the temporary bubble.

Figs. I and 2 show the open cup with the vertically projected cylindrical sheet of water and its breakings edge. In Fig. 3 the capillary tension has pulled in the

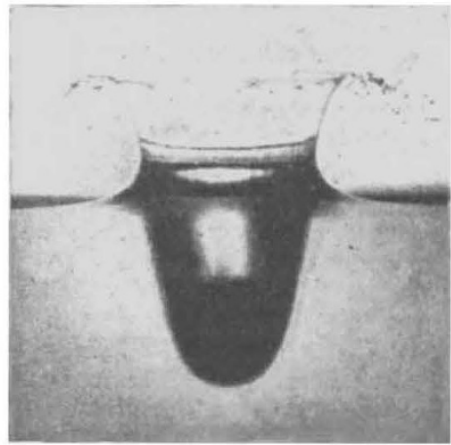

FiG. I. - -006s

sides, and is on the point of completing the bubble. In Fig. 4 the surface of the bubble is being pulled down by the descent of the ball, which reduces the pressurc within the bubble. As there is less mass in the film above than in the continuous liquid sides, the roof is at first drawn downwards. At this stage it also thickens from an influx of liquid from the sides, or appears to do so, and this influx, meeting in the centre, has not come perfectly to rest so that a musical note is sounded at each fall, the variations in the tone being surpirisingly large and erratic. A record of the note,

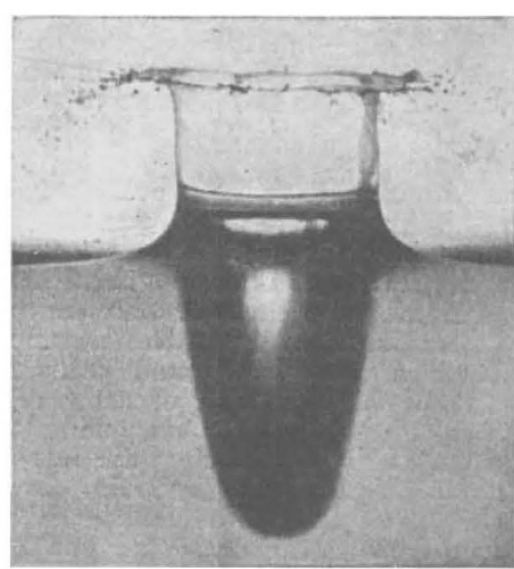

FiG. 2:-0.008s.

if it could be kept audible by acoustically screening off the snap of the spark, and a comparison of this with the alternating periods revealed by the photographs, should, if it were worth while, make clear the source of the musical note and the cause of its variation.

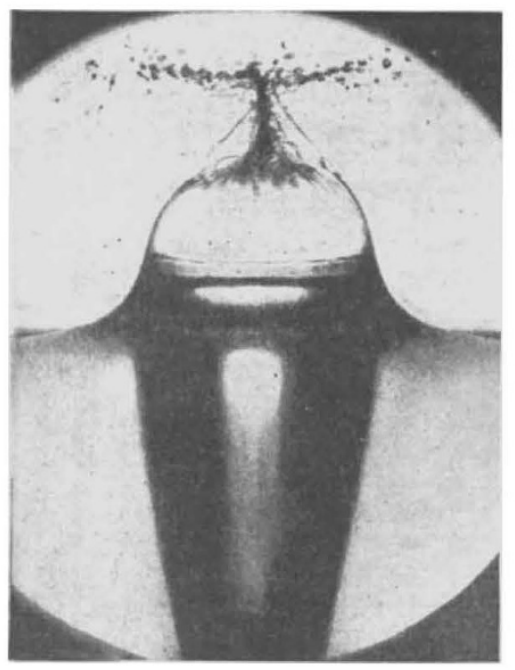

FIG. 3.-0.015s.

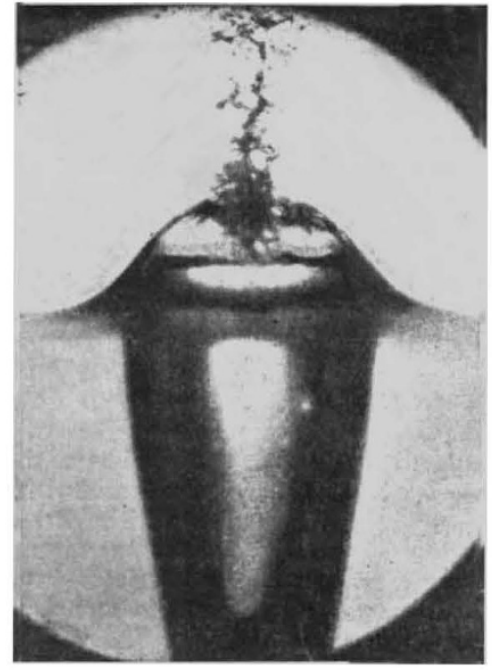

FIG. 4.-0.021S

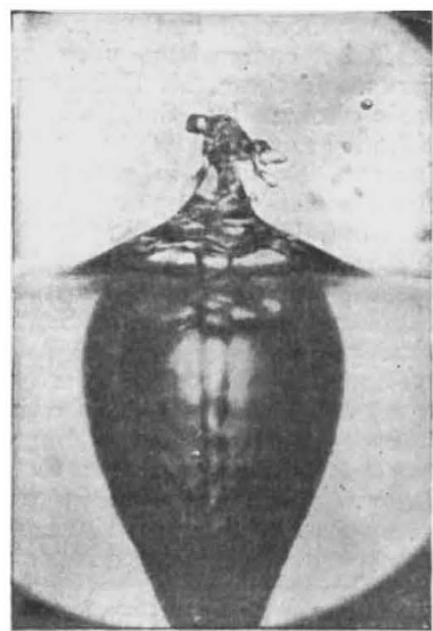

FIG. 5.-0.057s.

Rough spt.ere falling $x_{4} 0 \mathrm{~cm}$, into water. From "A Study of Splashes."

gives rise to a powerful descending jet seen in Figs. 5 to I I of the book. The acceleration is greater than that due to gravity, and it appears that the curved path of the inrushing liquid concave downwards enables it to balance the excess of atmospheric pressure above the bubble.

It would be interesting to see a number of photographs of drops falling into water in conditions often
It is not possible in the space available to illustrate or describe more of the results shown in Prof. Worthington's interesting book. It is hoped that enough has been said to excite the interest of experimentalists and others, who will be well repaid by a study of the original, which should find a place in every scientific library.

$$
\text { C. V. Bors. }
$$
No. 2.335 , roL. 781 